\title{
Congenital rubella syndrome despite maternal antibodies
}

\begin{abstract}
THE CASE: In January 2004 a female infant was delivered by cesarean section at 36 weeks' gestation because of severe intrauterine growth restriction. The mother was a healthy 31 -year-old gravida 4 para 2 woman who had been travelling in Sri Lanka from before conception until 6 weeks' gestation. Blood drawn for antenatal tests at 6 weeks' gestation upon her return to Canada was not initially tested for rubella titre. A rubella titre at 12 weeks' gestation $(425 \mathrm{IU} / \mathrm{mL})$ was interpreted as indicating immunity. The pregnancy was uncomplicated, without a history of fever or rash.

The mother had immigrated to Canada from Sri Lanka in 1993, at which point her rubella titre had been $17 \mathrm{IU} / \mathrm{mL}$. She did not recall a history of rubella infection or of rubella vaccination. She had delivered a healthy child in 1996, but rubella screening had not been requested during that pregnancy. In her other 2 pregnancies, which did not carry to term, her rubella titre had not been tested.

Findings on initial physical examination of the daughter born in 2004 were unremarkable. Her birth weight was $1.58 \mathrm{~kg}$, head circumference $28.3 \mathrm{~cm}$ and length $41 \mathrm{~cm}$ (all below the third percentile). Hematologic studies revealed a normal leukocyte count and hemoglobin concentration but a low platelet count $\left(49 \times 10^{\circ} / \mathrm{L}\right)$. During the first few days, the infant had hypoglycemia, hypocalcemia and hyponatremia, with a single seizure being attributed to hyponatremia. Ultrasonography of the head yielded normal findings. A murmur was noted on day 3 of life, and echocardiography demonstrated a large patent ductus arteriosis, a patent foramen ovale and severe pulmonary valvular stenosis. The infant was subsequently given a diagnosis of bilateral congenital cataracts.

On the first day of the infant's life, her rubella IgG titre was $243 \mathrm{IU} / \mathrm{mL}$ (immunity is indicated at a titre of $10-15 \mathrm{IU} / \mathrm{mL}$ or greater) and the rubella IgM titre was 1.123 index units (a positive result is 0.3 or more index units [Behring Enzygnost, Behring Diagnostics, Marburg, Germany]). Rubella virus was cultured from the urine and from both extracted cataracts.

After delivery, the stored antenatal serum that had been collected at 6 weeks' gestation was tested, and the rubella $\operatorname{IgG}$ and $\operatorname{IgM}$ titres were both positive $(281 \mathrm{IU} / \mathrm{mL}$ and 0.619 index units respectively). All rubella titres were performed at the Ontario Central Public Health Laboratory.
\end{abstract}

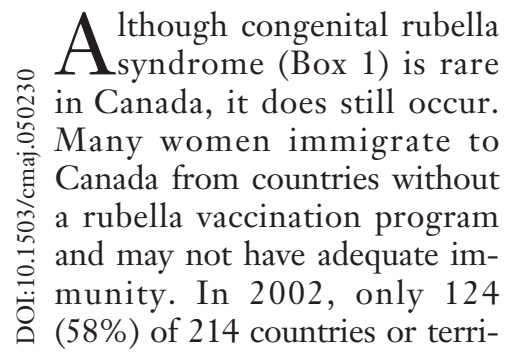

\section{Key points}

- Consider rubella vaccination of nonpregnant immigrant women at their first encounter with the health care system.

- Women with a low positive IgG titre (e.g., 10$15 \mathrm{IU} / \mathrm{mL}$ ) before pregnancy may benefit from repeat rubella vaccination.

- Women should be screened for rubella susceptibility at each pregnancy, because immunity (from previous vaccination or natural immunity) can wane.

- If a pregnant woman with a low positive IgG titre has recently been exposed to rubella, measurement of her IgM titre can help distinguish between new infection and immunity.

- Seronegative pregnant women need to be given rubella vaccine after delivery. tories around the world had implemented a rubella vaccination program. ${ }^{1}$ An Australian study found that women born in Asia, sub-Saharan Africa and South America were 5 times as likely as other women to be seronegative for rubella virus. In a review of all cases of infants with congenital rubella syndrome in the United States reported to the National Congenital Rubella Syndrome Registry from 1997 to $1999,83 \%(20 / 24)$ were born to Hispanic mothers and $91 \%$ (21/23) were born to foreignborn mothers.

Sri Lanka does not have a rubella vaccination program; therefore, if our patient's low positive titre from 1993 was a true-positive result, it was likely the result of natural immunity. Since serologic testing for rubella was documented only once preceding this pregnancy, we strongly suspect, but cannot confirm, that this case of congenital rubella syndrome resulted from rubella reinfection: the IgG titre had increased markedly, from $17 \mathrm{IU} / \mathrm{mL}$ in 1993 to $281 \mathrm{IU} / \mathrm{mL}$ 10 years later, with a positive IgM titre and no history of revaccination. A rubella-specific IgM test performed at 12 weeks' gestation would have confirmed infection rather than immunity.

Numerous cases of congenital rubella syndrome due to rubella reinfection have been reported. ${ }^{2}$ All involved exposure to rubella in the first trimester of pregnancy after both natural and vaccine-induced immunity, usually with subclinical infection in the mother. The immune response may involve more than humoral immunity, but it is clear that rubella antibodies decline over time and may increase the risk of reinfection. In a study involving Korean children, $18.8 \%$ of those who had been vaccinated and $13.8 \%$ of those with natural immunity were found to be seronegative for rubella virus after 3 years. An 
Italian study showed that $9.8 \%$ of vaccinated girls were reinfected by wild-type rubella virus within 5 years.

Prenatal serologic testing for rubella usually includes only the IgG titre, and the result is reported as "immune" or "nonimmune" (Box 2). There is controversy over what level of titre confers immunity. Bullens and coauthors ${ }^{2}$ documented that 8 of 16 mothers whose infants had congenital rubella syndrome had rubella IgG titres of at least $15 \mathrm{IU} / \mathrm{mL}$ at the start of pregnancy, a level considered to be protective. Their finding questions whether the current cutoff level $10-15 \mathrm{IU} / \mathrm{mL}$ is too low to be considered protective and whether women with low positive rubella titres before pregnancy might benefit from repeat vaccination.
In the absence of quantitative IgG reporting, clinicians do not know which women have low positive titres. A further problem is that a single IgG titre without an IgM titre cannot differentiate recent infection from immunity, as demonstrated by the case we have reported. Processing all rubella serologic tests in a centralized laboratory with quantitative IgG titres may enable the detection of significant rises in IgG levels in subsequent samples. Rubella-specific IgM titres could be routinely measured during the prenatal screening, but the cost-effectiveness of this approach would be questionable.

Given that more than half of the world's population lives in countries without a rubella vaccination program, many new Canadians are susceptible to rubella. Physicians should con-

\section{Box 1: Clinical features of congenital rubella syndrome}

\section{Classic triad}

- Congenital heart disease (e.g., patent ductus arteriosis, pulmonary artery stenosis, pulmonary valvular stenosis)

- Ocular defects (e.g., congenital cateracts, microphthalmos, pigmentary retinopathy, congenital glaucoma)

- Hearing loss

Congenital rubella syndrome is usually associated with a failure to thrive and developmental delay as well as microcephaly. Other common presentations at birth include:

- purpuric rash

- hepatosplenomegaly

- meningoencephalitis

- radiolucent bone

- hepatitis

- thrombocytopenia

\section{Box 2: Limitations of antenatal screening for rubella}

- Laboratories usually report result of serologic test for IgG titre as "immune" (> 10-15 IU/mL*) or "nonimmune"

- Low or borderline lgG levels are not detected

- Reinfection resulting in congenital rubella syndrome has occurred at low levels of immunity

- Substantial rises in IgG levels (indicating possible infection) cannot be detected without quantitative reporting

- Measurement of the $\lg M$ titre is necessary to distinguish between immunity and infection

*Laboratories using the Abbott AxSym immunoassay system (Abbott Laboratories Canada, Mississauga, Ont.) consider a positive result to be $10 \mathrm{IU} / \mathrm{mL}$ or greater; most other laboratories consider a positive result to be $15 \mathrm{IU} / \mathrm{mL}$ or greater. sider offering measles-mumpsrubella vaccination to nonpregnant immigrant women from developing countries at their first contact with the health care system. ${ }^{3}$ Serologic screening before vaccination is not recommended and may result in a missed opportunity to vaccinate if pregnancy occurs in the interim or if the woman is lost to follow-up. Consultations before travel may represent an opportunity to review rubella immunization status of women of childbearing age.

Because rubella antibodies wane over time, screening for rubella susceptibility is recommended at the first prenatal visit for all pregnant women in Canada. In a Canadian study, $2.3 \%-13.3 \%$ of pregnant women were seronegative for rubella virus. A Quebec study found that only half of the $8.4 \%$ women who were rubella seronegative received the vaccine after delivery. Standing orders for rubella vaccination after delivery may increase immunization rates.

\section{Anna Banerji}

Public Health Sciences

University of Toronto

Department of Pediatrics

St. Joseph's Health Centre

Toronto, Ont.

Elizabeth Lee Ford-Jones

Division of Infectious Diseases

Hospital for Sick Children

Toronto, Ont.

Edmond Kelly

Department of Pediatrics

Mount Sinai Hospital

Toronto, Ont.

Joan Louise Robinson

Department of Pediatrics

Stollery Children's Hospital

Edmonton, Alta.

\section{References}

1. Robertson SE, Featherstone DA, Gacic-Dobo M, Hersh BS. Rubella and congenital rubella syndrome: global update. Rev Panam Salud Publica 2003;14(5):306-15.

2. Bullens D, Smets K, Vanhaesebrouck $\mathrm{P}$. Congenital rubella syndrome after maternal reinfection. Clin Pediatr 2000;39(2):113-6.

3. Ford-Jones EL, Tam T, Kelly E, DeSerres G. Prevention of congenital rubella syndrome. Statement of the Infectious Disease and Immunization Committee, Canadian Pediatric Society. Paediatr Child Health 1999;4(2):155-7. 